\title{
Solute effects on supercritical fluid
}

\author{
$\operatorname{AUTHOR}(\mathrm{S})$ :
}

Yamamoto, Takahiro; Matsumoto, Mitsuhiro

\section{CITATION:}

Yamamoto, Takahiro ...[et al]. Solute effects on supercritical fluid. Molecular Simulation 2011, 37(13): 1091-1096

\section{ISSUE DATE:}

2011-08-12

URL:

http://hdl.handle.net/2433/197331

\section{RIGHT:}

The Version of Record of this manuscript has been published and is available in Molecular Simulation (2011) http://www.tandfonline.com/ 10.1080/08927022.2011.582104; この論文 は出版社版でありません。引用の際には出版社版をご確認ご利用ください。; This is not the published version. Please cite only the published version. 


\section{Solute Effects on Supercritical Fluid}

\section{Takahiro Yamamoto* and Mitsuhiro Matsumoto}

Department of Mechanical Engineering and Science,

Graduate School of Engineering, Kyoto University, Kyoto 606-8501, Japan

E-mail: wish-on-a-star@t05.mbox.media.kyoto-u.ac.jp

Tel: $+81-75-753-5217$

Fax: $+81-75-771-7286$

(Received on July 30, 2010; final version received on April 2, 2011)

Supercritical carbon dioxide has been widely used as solvents, although its solubility is much lower than that of typical organic solvents of liquid state in general. Entrainer effects are known and utilized, in which adding small molecules, such as alcohols and hydrocarbons, increase the solubility and the chemical reactivity. In this study, we investigated the change of local fluid structure and thermodynamic properties with molecular dynamics simulations, focusing on the entrainer effect. A monatomic fluid mixture model based on the Lennard-Jones model potential was adopted for simplicity, the parameters of which roughly corresponds to those of carbon dioxide (solvent) and ethanol (solute). A series of simulations were performed in the vicinity of the gas-liquid 
critical point. The radial distribution function and the coordination number reveal the enhancement of the solvent local density. The solvent chemical potential was evaluated with the Widom's particle insertion method. The chemical potential change by adding solutes is smallest in magnitude at the critical point, qualitatively agreeing with recent experimental analysis and interpretation with a small-angle neutron scattering method.

Keywords: supercritical fluid, entrainer effect, chemical potential, molecular simulation

\section{Introduction}

Supercritical fluids (SCFs) are widely used in chemical engineering [1, 2]. One of the reasons is the controllability of their density in a wide range due to the divergently large compressibility in the vicinity of their gas-liquid critical point (CP). As a solvent, however, SCFs are poor in general, when compared with conventional solvents in liquid state, because the density of SCFs is lower than that of normal liquids. Cosolvents, or entrainers, are proposed $[1,2]$ to improve the solvent property. For example, carbon dioxide is a widely used SCF solvent in "green chemistry", because of its mild conditions of the $\mathrm{CP}$, its safety, and its high cost performance. When a tiny amount of hydrocarbons, alcohols, or water is added, the solubility of various organic compounds is drastically enhanced.

Concerning the mechanism of such entrainer effects, much attention has been paid to microscopic structure in SCF mixtures; see [3] and references therein. In a rough sketch, the added entrainer molecules enhance the local density inhomogeneities by attracting solvent around them. 
Experiments to directly investigate the static and dynamic characters of the local structures were reported, with small-angle X-ray scattering [4, 5], Raman spectrum [6], dynamic light scattering [7], and small-angle neutron scattering (SANS) $[8,9]$. Recently, Sato et al. reported the structure of SCF carbon dioxide with alcohols with the SANS techniques $[10,11]$, where pressure dependence of the correlation length and the intensity were analyzed from the viewpoints of density fluctuations. They proposed that the third moment of the density fluctuations, or the skewness $\gamma$, is a good indicator of the local density distribution, and derived the relation between $\gamma$ and the chemical potential of solvent $\mu_{v}$ [9]. Interestingly, they concluded that $\mu_{v}$ increases in the vicinity of the CP by adding entrainers [11], which is contrary to a naive argument of osmotic pressure, where $\mu_{v}$ is expected to be a decreasing function of solute concentration.

In this paper, we report molecular dynamics simulations of model SCF systems, focusing mainly on the change of thermodynamic quantities by adding solute. Since the pioneering work of Petsche and Debenedetti [12], a large number of studies with molecular dynamics (MD) or Monte Carlo (MC) simulations were reported on the solute effects on SCF, but most of them are focused on the local density and clustering behaviour around the solute molecules. Here, we try to figure out the local density and thermodynamic properties of solvents..

\section{Simulation Method}

\subsection{System}

We carried out a series of canonical ensemble (NVT constant) MD simulations of monatomic fluid. The total number of particles $N$ is only 1,000 , close to [12]; the 


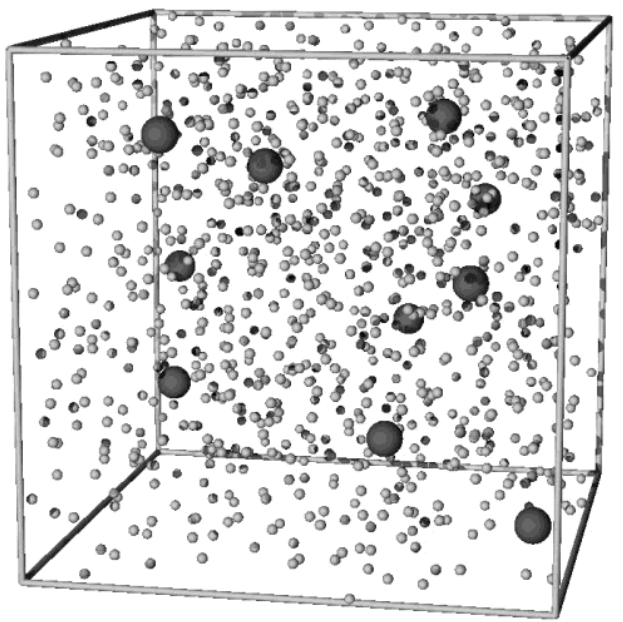

Fig. 1 Snapshot of LJ fluid with solute particles at the critical point. Note that the size ratio displayed here is exaggerated; the ratio in the simulation is $\sigma_{u} / \sigma_{v}=4.42 / 3.65=1.21$, based on the critical volume of carbon dioxide (solvent) and ethanol (solute).

system size is certainly too small to investigate long range fluctuations near the gasliquid critical point quantitatively, but will be sufficient to qualitatively elucidate the entrainer effects. We compare molecular structures and thermophysical properties between a pure solvent system and a mixture (990 solvent particles +10 solutes) at the same $(T, V)$ conditions, corresponding to the experimental conditions [10, 11], where the mole fraction of solute alcohol in SCF carbon dioxide is 0.01 .

In the MD simulations, the equations of motion are numerically integrated with the leapfrog algorithm. The velocity scaling technique is adopted so that the average temperature becomes a given value. Time average for equilibrium properties is taken for sufficiently long run at each condition.

A snapshot for the mixture is shown in Fig. 1, where the added solute particles are uniformly dispersed in general. 


\begin{tabular}{|c|c|c|c|}
\hline & & $\begin{array}{c}\text { Solvent } \\
\mathrm{CO}_{2}\end{array}$ & $\begin{array}{c}\text { Solute } \\
\mathrm{C}_{2} \mathrm{H}_{5} \mathrm{OH}\end{array}$ \\
\hline \multicolumn{2}{|c|}{ Critical temperature $T_{c}(\mathrm{~K})$} & 304 & 514 \\
\hline \multicolumn{2}{|c|}{ Critical pressure $P_{c}(\mathrm{MPa})$} & 7.38 & 6.14 \\
\hline \multicolumn{2}{|c|}{ Critical density $\rho_{c}\left(\mathrm{~kg} / \mathrm{m}^{3}\right)$} & 468 & 276 \\
\hline \multicolumn{2}{|c|}{ Critical volume $V_{c}\left(\mathrm{~m}^{3} / \mathrm{mol}\right)$} & $9.40 \times 10^{-5}$ & $16.7 \times 10^{-5}$ \\
\hline \multicolumn{2}{|c|}{ Molecular mass } & 44.0 & 46.1 \\
\hline $\begin{array}{c}\text { Model } \\
\text { parameters }\end{array}$ & $\begin{array}{c}\text { Energy } \varepsilon(\mathrm{J}) \\
\text { Diameter } \sigma(\mathrm{m}) \\
\text { Mass } m(\mathrm{~kg})\end{array}$ & $\begin{array}{l}3.20 \times 10^{-21} \\
3.65 \times 10^{-10} \\
7.31 \times 10^{-26}\end{array}$ & $\begin{array}{l}5.40 \times 10^{-21} \\
4.42 \times 10^{-10} \\
7.64 \times 10^{-26}\end{array}$ \\
\hline
\end{tabular}

\subsection{Interaction model}

As the solvent-solvent interaction, we adopt the Lennard-Jones (LJ) 12-6 potential for simplicity:

$$
\phi_{v}(r)=4 \varepsilon_{v}\left[\left(\frac{\sigma_{v}}{r}\right)^{12}-\left(\frac{\sigma_{v}}{r}\right)^{6}\right]
$$

where $r$ is a distance between solvent particles, $\sigma_{v}$ and $\varepsilon_{v}$ are the LJ parameter of size and energy, respectively.

The solute is also modelled as a monatomic LJ fluid. Since we are interested in ethanol as the typical entrainer, the $\mathrm{LJ}$ parameters $\sigma_{u}$ and $\varepsilon_{u}$ are chosen so that the critical properties correspond to those of ethanol. Based on Table 1, we choose

$$
\sigma_{u} / \sigma_{v}=1.21, \varepsilon_{u} / \varepsilon_{v}=1.69
$$

The Lorentz-Berthelot combination rule is adopted so that the parameters of interaction between solute and solvent are

$$
\sigma_{u v}=\frac{\sigma_{u}+\sigma_{v}}{2}=1.105 \sigma_{u}, \quad \varepsilon_{u v}=\sqrt{\varepsilon_{u} \varepsilon_{v}}=1.30 \varepsilon_{v}
$$




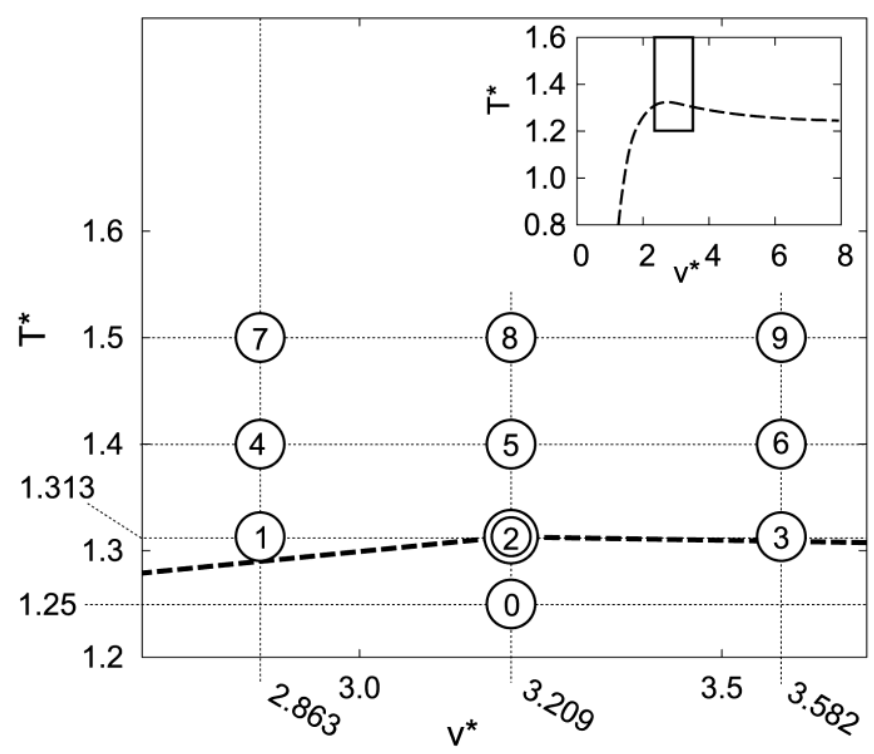

Fig. 2 Simulation conditions. The dashed curves roughly show the gas-liquid coexistence region. The condition 2 corresponds to the critical point.

As the solute-solvent interaction is stronger than that of solvent-solvent, we expect that the model works as an attractive solute [14].

In the following descriptions, we use reduced units with the solvent parameters $\sigma_{v}, \varepsilon_{v}$, and $m_{v}$ (particle mass), such as

$$
\text { temperature } T^{*}=\frac{k_{B} T}{\varepsilon_{v}} \text {, pressure } P^{*}=\frac{\sigma_{v}{ }^{3} P}{\varepsilon_{v}} \text {, volume } v^{*}=\frac{v}{\sigma_{v}{ }^{3}}
$$

The asterisk is often omitted to simplify the expression.

\subsection{Simulation conditions}

We focus on properties of fluid close to the gas-liquid critical point. To determine the critical point, a series of MD simulations for a single component (i.e., pure solvent) LJ system were carried out at temperature $1.0 \leq T \leq 1.6$ with various density; the particle interaction is truncated at $r=4.0$, and no tail correction is made. We fitted the obtained pressure data to a simple van der Waals equation of state to find the critical condition as 


$$
T_{c}=1.313, P_{c}=0.1536, \rho_{c}=0.3116, v_{c}=3.209
$$

which is within the range of reported values $\left(T_{c}=1.313 \sim 1.35, P_{c}=0.123 \sim 0.145\right.$, $v_{c}=2.857 \sim 3.257$ ) cited in Table 4 in [15], and close to a more recent work [16]. We selected 10 simulation conditions in the vicinity of $\left(T_{c}, v_{c}\right)$, as shown in Fig. 2. A cubic cell is used, the size of which ranges from 14.2 to $15.3 \sigma_{v}$.

We use the time step $0.001 \tau$, where $\tau$ is the unit time defined as $\sigma_{v} \sqrt{m_{v} / \varepsilon_{v}}$. The total steps of the MD simulation for each condition are 30,000, of which the initial 10,000 steps are discarded.

\section{Results}

\subsection{Structure change}

The solvent-solvent radial distribution function $g_{v v}(r)$ is calculated at each condition;

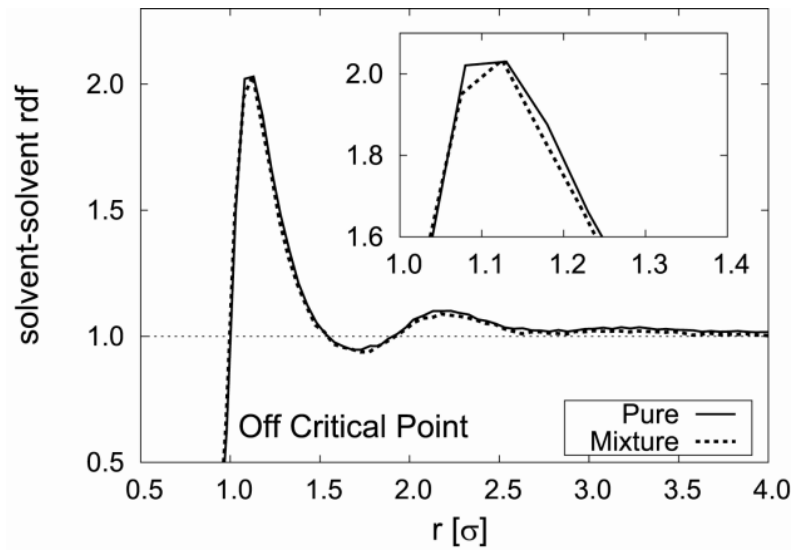

(a) $T^{*}=1.313$ and $v^{*}=2.863$ (cond. 1 )

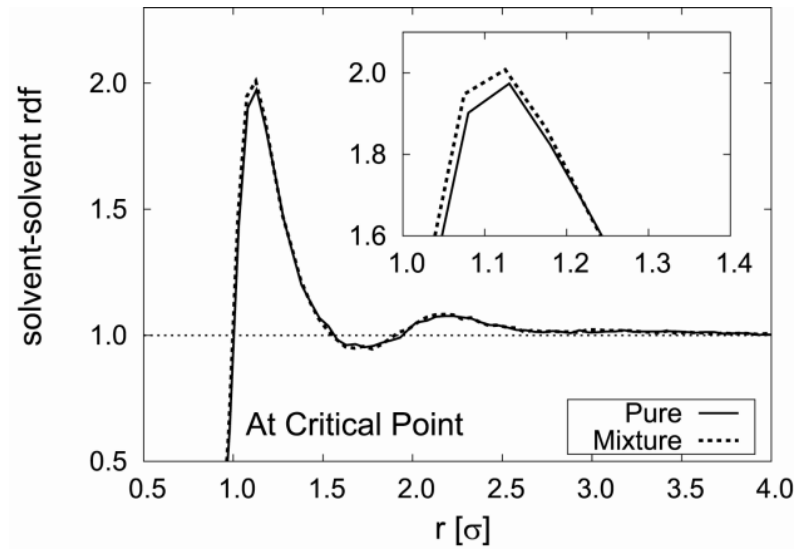

(b) $T^{*}=1.313$ and $v^{*}=3.209$ (cond. 2).

Fig.3 Radial distribution function between solvent molecules. At the critical point (b), addition of solute particles enhances the first peak. 
examples are shown in Fig. 3. In general, the first peak of $g_{v v}(r)$ is expected to slightly decreases by adding the solute particles mainly because the solvent density is lowered. At the $\mathrm{CP}$, however, the peak increases, which suggests that the solvent structure at the $\mathrm{CP}$ is easily affected by the small amount of solute; a similar enhancement of the $g_{v v}(r)$ peak is observed in Conditions 5-7, although the degree is very small.

The solvent-solvent coordination number $N_{v v}$ is examined to see the change:

$$
N_{v v}=4 \pi n_{v} \int_{0}^{r_{\min }} g_{v v}(r) r^{2} d r
$$

where $n_{v}$ is the number density of solvent, and the integration is done up to the first minimum of $g_{v v}(r)$. The obtained $N_{v v}$ is $6.6^{-8.4}$ depending on the condition. We focus on the change $\Delta N_{v v}=N_{v v}$ (mixture) $-N_{v v}$ (pure), which is shown in Table 2. Although the tendency is not so clear, the change is positive at the critical point (and several other conditions); note that we would have $\Delta N_{v v} \approx-0.01$ due to the decrease of $n_{v}$ if there is no shape change in $g_{v v}(r)$. These results indicate the augmentation of solvent local density by solute [3], although its magnitude is very small.

\subsection{Chemical potential}

The chemical potential is calculated with a standard technique of Widom's particle insertion [17],

$$
\mu^{\text {total }}=\mu^{i d}+\mu^{e x}
$$

where the first term is the ideal gas part,

$$
\mu^{i d}=-k_{B} T \ln \left\langle\left(\frac{2 \pi m k_{B} T}{h^{2}}\right)^{\frac{3}{2}} \cdot \frac{V}{N}\right\rangle
$$


The second represents the excess (or residual) part due to particle interactions,

$$
\mu^{e x}=-k_{B} T \ln \left\langle\exp \left[-\frac{\phi_{N+1}\left(\vec{r}_{1}, \vec{r}_{2}, \cdots, \vec{r}_{N}\right)}{k_{B} T}\right]\right\rangle
$$

where \langle\rangle is evaluated as an statistical average of the Boltzmann factor of a randomly inserted particle, the potential energy of which is $\phi_{N+1}$. It would be better to evaluate the chemical potential in the NPT ensemble [18] instead of the canonical one because the constant volume condition suppresses the long-range fluctuations. In this study, however, we investigate the density dependence of $\mu$ by adopting the canonical ensemble.

In Table 2, the change $\Delta \mu_{v}=\mu_{v}$ (mixture $)-\mu_{v}$ (pure) for the solvent is shown.

As expected, $\Delta \mu_{v}^{\text {total }}$ is negative for all conditions, which agrees with the osmotic

Table 2 Change of thermodynamic properties, (mixture)-(pure). The temperature $T^{r}$ and the specific volume per particle $v^{r}$ are relative values to the $\mathrm{CP}$ (gas-liquid critical point). The difference of energy $\Delta E$, Gibbs free energy $\Delta G$, the enthalpy $\Delta H$, and entropy $\Delta S$ are for 1000 particle systems. Thermodynamic quantities are all expressed in reduced units. The underlines indicate the extremum among the ten conditions.

\begin{tabular}{ccc|rcrrrrrr}
\hline $\begin{array}{c}\text { Condition } \\
\text { no. }\end{array}$ & $T^{r}$ & $v^{r}$ & \multicolumn{1}{c|}{$\Delta N_{v}$} & $\Delta E$ & $\begin{array}{c}\Delta P \\
\left(\times 10^{-3}\right)\end{array}$ & \multicolumn{1}{c}{$\Delta \mu_{v}^{e x}$} & \multicolumn{1}{c}{$\Delta \mu_{v}^{\text {total }}$} & $\begin{array}{c}\Delta G \\
\left(\times 10^{-2}\right)\end{array}$ & \multicolumn{1}{c}{$\Delta H$} & $\Delta S$ \\
\hline 0 & 0.952 & 1.000 & 0.029 & -29.45 & -2.06 & -0.035 & -0.048 & -1.31 & -36.1 & 75.8 \\
\hline 1 & 1.000 & 0.892 & -0.072 & -23.35 & 0.22 & -0.037 & -0.050 & -1.34 & 24.0 & 120.3 \\
$2(\mathrm{CP})$ & 1.000 & 1.000 & 0.040 & $\underline{-63.44}$ & 2.21 & $\underline{-0.006}$ & $\underline{-0.019}$ & $\underline{-1.05}$ & -56.4 & $\underline{36.5}$ \\
3 & 1.000 & 1.116 & -0.032 & -5.31 & 2.58 & -0.016 & -0.029 & -1.13 & 3.9 & 87.3 \\
\hline 4 & 1.066 & 0.892 & -0.025 & -36.06 & 7.74 & -0.031 & -0.045 & -1.35 & -13.9 & 88.3 \\
5 & 1.066 & 1.000 & 0.040 & 6.08 & 1.02 & -0.020 & -0.034 & -1.22 & 9.4 & 93.6 \\
6 & 1.066 & 1.116 & $\underline{0.080}$ & -35.29 & $\underline{-8.58}$ & -0.016 & -0.030 & -1.16 & $\underline{-66.0}$ & 38.0 \\
\hline 7 & 1.142 & 0.892 & -0.002 & -12.07 & 3.50 & -0.024 & -0.039 & -1.32 & -2.1 & 88.4 \\
8 & 1.142 & 1.000 & -0.022 & -14.13 & -3.58 & -0.021 & -0.036 & -1.27 & -25.6 & 66.7 \\
9 & 1.142 & 1.116 & -0.020 & 11.87 & -4.31 & -0.013 & -0.028 & -1.18 & -3.6 & 76.2 \\
\hline
\end{tabular}


pressure argument. It is interesting, however, that the absolute values of both $\Delta \mu_{v}^{e x}$ and $\Delta \mu_{v}^{\text {total }}$ are smallest at the critical point; this behaviour is qualitatively similar to that reported in [11]. The origin of the smallest $\left|\Delta \mu_{v}^{\text {total }}\right|$ at the CP is not straightforward, but should be explained in terms of interaction term $\Delta \mu_{v}^{e x}$, as $\Delta \mu_{v}^{i d}$ has no density dependence.

\subsection{Other thermodynamic properties}

Similarly to $\mu_{v}$, the chemical potential of solute $\mu_{u}$ is easily evaluated; the results are not included in Table 2 because no specific tendency is observed, although a slight difference in volume dependence is discernible at the $\mathrm{CP}$. With these two $\mu$, we estimate the Gibbs free energy of the simulated system (1000 particles) as

$$
G(\text { pure })=1000 \mu_{\mathrm{v}}(\text { pure })
$$

and

$$
G(\text { mixture })=990 \mu_{\mathrm{v}}(\text { mixture })+10 \mu_{u}
$$

The change $\Delta G=G$ (mixture $)-G($ pure $)$ is shown in Table 2 . Since we obtain the total energy $E$ and the pressure $P$ directly in each simulation, other thermodynamic quantities are easily estimated. Especially important is the entropy,

$$
S=\frac{E+P V-G}{T}
$$

where $V$ is the volume of the system. In Table 2, the difference $\Delta S$ is shown along with $\Delta E, \Delta P, \Delta G$, and the enthalpy change $\Delta H$.

Naturally, the solute particles energetically stabilize the system at the $\mathrm{CP}$, as indicated in $\Delta E$. However, the pressure change $\Delta P$ seems to have no particular trends; the difference is much smaller than the pressure itself (e.g., $P=0.153$ and 
Table 3 Change of solvent chemical potential $\Delta \mu_{v}^{\text {total }}$, depending on the interaction parameter.

\begin{tabular}{c|l|ccc}
\hline \multicolumn{2}{l|}{} & $\begin{array}{c}v^{*}=2.863 \\
\text { (cond. 1) }\end{array}$ & $\begin{array}{c}v^{*}=3.209 \\
\text { (cond. 2, CP) }\end{array}$ & $\begin{array}{c}v^{*}=3.582 \\
\text { (cond. 3) }\end{array}$ \\
\hline \multirow{4}{*}{$T^{*}=1.313$} & $\varepsilon_{u} / \varepsilon_{v}=1.69, \varepsilon_{u v} / \varepsilon_{v}=1.30$ & -0.050 & -0.019 & -0.029 \\
& $\varepsilon_{u} / \varepsilon_{v}=1.85, \varepsilon_{u v} / \varepsilon_{v}=1.36$ & -0.043 & -0.021 & -0.031 \\
& $\varepsilon_{u} / \varepsilon_{v}=2.00, \varepsilon_{u v} / \varepsilon_{v}=1.41$ & -0.044 & -0.029 & -0.040 \\
\hline
\end{tabular}

$\Delta P=0.002$ at the $\mathrm{CP}$ ), and the attractive character of the solute, by which we expect $\Delta P \approx\left(\frac{\partial P}{\partial x}\right)_{T, V} \Delta x<0(x:$ composition of the solute $)$, is not clear. The canonical ensemble (constant V) may affect the results. From $\Delta S$, we speculate that the local density augmentation suppresses the entropy increase. Note that the condition 6 $\left(T^{r}=1.066\right.$ and $\left.\rho^{r}=1.116\right)$ is almost as singular as the $\mathrm{CP}$, from the viewpoints of thermodynamic properties as well as the local structure $\left(g_{v v}(r)\right.$ and $\left.\Delta N_{v v}\right)$.

\subsection{Dependence on the interaction parameter}

Assumption of the Lorentz-Berthelot combination rule is certainly too naive for the complex interaction between $\mathrm{CO}_{2}$ and ethanol. Results will strongly depend on the interaction, especially the parameter $\varepsilon_{u v}$. Thus we change $\varepsilon_{u v}$ and re-calculate the chemical potential change $\Delta \mu_{v}^{\text {total }}$; we still assume the Lorentz-Berthelot rule, but the solute-solute interaction is irrelevant due to the low concentration. Results are shown in Table 5 at $T^{*}=1.313$. Some dependence is certainly observed, but the tendency is similar; the chemical potential change is smallest at the critical point. 


\section{Conclusion}

We investigated the local fluid structure and the thermodynamic properties of a model supercritical fluid with molecular dynamics simulation technique, focusing on entrainer effects from solvent viewpoints. By adding a small amount of attractive solute, (i) solvent molecules slightly gather at the critical point (CP) while they tend to disperse off the $\mathrm{CP}$, and (ii) the chemical potential of solvent generally decreases in accordance with the osmotic pressure argument, but the change is the smallest at the $\mathrm{CP}$, the behaviour of which qualitatively agrees with the analysis on recent SANS experiments. We speculate that theses two closely relates to the mechanism of entrainer effects. Certainly the simulation system is too small to realize the long range fluctuations near the $\mathrm{CP}$, and the interaction model is too simple to incorporate realistic molecular interactions. Based on these findings, we will investigate the solubility change and other entrainer effects in more detail, using larger scale simulations and liquid theories.

\section{Acknowledgement}

This work was inspired by neutron scattering experiments of Dr. T. Sato, Prof. M. Sugiyama, and their co-workers. The authors are grateful to Prof. M. Sugiyama at Kyoto University for stimulating discussions over their recent experiments and incessant encouragement.

\section{References}

[1] Y. Arai, T. Sako, and Y. Takebayashi (eds.), Supercritical fluids: Molecular interactions, physical properties and new applications, Springer, Berlin, 2002.

[2] G. Brunner (ed.), Supercritical fluids as solvents and reaction media, Elsevier, 
Amsterdam, 2004.

[3] S. C. Tucker, Solvent density inhomogeneities in supercritical fluids, Chem. Rev., 99 (1999) pp. 391-418.

[4] K. Nishikawa, I. Tanaka, and Y. Amemiya, Small-angle X-ray scattering study of supercritical carbon dioxide, J. Phys. Chem., 100 (1996) pp. 418-412.

[5] K. Nishikawa, A. A. Arai, and T. Morita, Density fluctuation of supercritical fluids obtained from small-angle X-ray scattering experiment and thermodynamic calculation, J. Supercritical Fluids, 30 (2004) pp. 249-257.

[6] K. Saitow, K. Otake, H. Nakayama, K. Ishii, and K. Nishikawa, Local density enhancement in neat supercritical fluid due to attractive intermolecular interactions, Chem. Phys. Lett., 368 (2003) pp. 209-214.

[7] K. Saitow, D. Kajiya, and K. Nishikawa, Time evolution of density fluctuation in supercritical region. I. Non-hydrogen-bonded fluids studied by dynamic light scattering, J. Phys. Chem. A, 100 (2005) pp. 83-91.

[8] T. Sato, M. Sugiyama, M. Misawa, S. Takata, T. Otomo, K. Itoh, K. Mori, and T. Fukunaga, A new analysing aproach for the structure of density fluctuation of supercritical fluid, J. Phys.: Cond. Matt., 20 (2008) 104203.

[9] T. Sato, M. Sugiyama, K. Itoh, K. Mori, T. Fukunaga, M. Misawa, T. Otomo, and S. Takata, Structural difference between liquidlike and gaslike phases in supercritical fluid, Phys. Rev. E, 78 (2008) 051503.

[10] T. Sato, M. Sugiyama, M. Misawa, K. Hamada, K. Itoh, K. Mori, and T. Fukunaga, Structural investigation on supercritical carbon dioxide and its mixture with alcohol, J. Mol. Liq. 147 (2009) pp. 102-106.

[11] T. Sato, Structural investigation on supercritical carbon dioxide, $\mathrm{PhD}$ thesis, Kyoto University (2009). 
[12] I. B. Petsche and P. G. Debenedetti, Solute-solvent interactions in infinitely dilute supercritical mixtures: A molecular dynamics investigation, J. Chem. Phys. 91 (1989) pp. 7075-7084.

[13] B. E. Poling, J. M. Prausnitz, and J. P. O’Connell, The properties of gases and liquids, $5^{\text {th }}$ ed, McGraw-Hill, New York, 2000.

[14] A. A. Chialvo and P. T. Cummings, Solute-induced effects on the structure and thermodynamics of infinitely dilute mixtures, AIChE J., 40 (1994) pp. 1558-1573.

[15] J. Kolafa and I. Nezbeda, The Lennard-Jones fluid: an accurate analytic and theoretically-based equation of state, Fluid Phase Equil., 100 (1994) pp. 1-34.

[16] J. M. Caillol, Critical-point of the Lennard-Jones fluid: A finite-size scaling study, J. Chem. Phys., 109 (1998) pp. 4885-4893.

[17] B. Widom, Potential-distribution theory and the statistical mechanics of fluids, J. Phys. Chem., 86 (1982) pp. 869-872.

[18] K. S. Shing and S. T. Chung, Computer simulation methods for the calculation of solubility in supercritical extraction systems, J. Phys. Chem., 91 (1987) pp. 1674-1681. 